\title{
Artificial Neural Network Modelling for the Removal of Fe (III) from Aqueous Solutions Using Chitosan Magnetite Nano Composites (CMNs)
}

\author{
Mini Namdeo ${ }^{1^{*}}$, Rama Mehta ${ }^{2}$, Mehta VK$^{3}$ and Vijaya Agarwala ${ }^{1}$ \\ ${ }^{1}$ Centre of Excellence-Nanotechnology, Indian Institute of Technology, Roorkee, Uttarakhand, India \\ ${ }^{2}$ National Institute of Hydrology, Roorkee, Uttarakhand, India
}

${ }^{3}$ College of Engineering Roorkee, Roorkee, Uttarakhand, India

\begin{abstract}
A two-layer Artificial Neural Network (ANN) model was engendered to soothsay the deliberation efficacy of Fe (II) particles from fluid arrangement utilizing chitosan magnetite nano composites (CMNs). The sorbet stock arrangement was yare by dissolving a pre-computed amount of $\mathrm{FeCl}_{3}$ in twofold refined water to give last fixation 100 $\mathrm{mgl}^{-1}$. The stock arrangement was debilitated to get standard arrangements with fixation in the scope of 5-30 mgl-1 and their last $\mathrm{pH}$ was transmuted in accordance with 4.5. Fifty millilitres of $\mathrm{FeCl}_{3}$ arrangement of fancied focus was put in a $125 \mathrm{ml}$ Erlenmeyer flagon containing $0.02 \mathrm{~g}$ of $\mathrm{CMN}$ sorbent. A period of 3 hours was discovered adequate to accomplish the balance. The ANN model was intended to suspect sorption efficacy of CMNs for target metal particle by amalgamating back spread (BP) with guideline segment examination. A sigmoid axon was utilized as exchange capacity for information and yield layer. The Levenberg-Marquardt calculation (LMA) was connected, giving a base estimation of mean squared mistake (MSE) for preparing and cross approbation at the 6th place of decimal.
\end{abstract}

Keywords: Artificial neural network; Chitosan; Fe (II) removal; Nano composites

\section{Introduction}

The contamination, on account of the propinquity of substantial metal particles, is an encompassing issue of overall premium. Iron is one of the imperative metals that is paramount for development and amendment of person. In any case, its overdose can prompt solemn medical issues, for example, anorexia, the runs, diphasic stun, metabolic acidosis and demise [1]. Furthermore, the patient may encounter vascular blockage of the gastrointestinal tract, cerebrum, spleen and thymus [2]. With excruciating iron harming, a great part of the harm to the gastrointestinal tract and liver may come about because of the high-inhibited iron fixation and free radical engenderment prompting hepatotoxicity by betokens of lipid peroxidation and extirpation of the hepatic mitochondria Thus of iron stockpiling infection, the liver moves toward becoming cirrhotic. Hematoma, an essential tumour of the liver, has turned into the well-kenned reason for death among patients with hemochromatosis [3]. Later past has visually perceived astronomically immense misuse of an assortment of sorbents for the compelling voidance of $\mathrm{Fe}$ (III) from modern effluents and ground dihydrogen monoxide [4-10]. As of tardy ecological scientific experts have concentrated their exploration on abusing biopolymers as proficient contrivance for partition of Fe (III) like metal particles due to their simple accessibility, ease, propinquity of an assortment of functionalities, their non-perilous nature, and so on [11]. Among different biopolymer accessible, chitosan has the most eminent sorption limit with regards to a few metal particles, conceivably because of propinquity of essential amine at C-2 position of the glucosamine build-ups [12].

This biosorbent has a few downsides like non-abrasiveness, inclination to agglomerate and to shape gels in fluid arrangement, lastly not all that promptly accessibility of responsive restricting destinations [13]. That is the reason that such a potential sorbent has been bound just to research centre tribulations. With a categorical end goal to beat these impediments and other operational challenges, we thus propose chitosan-magnetite nano composite (CMNs) as a novel potential sorbent for prosperous voidance of Fe (III) from watery arrangements. This recently engendered CMN has not just solid metal chelating propensity because of proximity of chitosan yet it likewise has extra element of nano materials like expansive surface region, scattered nature, opportunity from convectional segments of settled stuffed particles and inflexible layers. Furthermore, because of alluring nature of CMN, it can without much of a stretch be isolated from the sorption framework by utilizing alluring field of opportune quality. To accomplish an ideal administration for any control measure, the conception of demonstrating for a proficient operation and configuration ought to be engendered $[14,15]$. An astounding agent model can give a great answer for the procedure control. It is probably going to demystify the genuine procedure execution building up a nonstop control technique for such kind of advances. In view of dependable, vigorous and eminent qualities in catching the non-direct connections of factors in involute frameworks, utilizations of Artificial Neural Network (ANN) have been efficaciously utilized in ecological building [16-18].

Alongside voidance of poisonous metals utilizing chitosan magnetite nano composites from waste dihydrogen monoxide [15], this paper portrays a two-layer ANN exhibit utilizing a back spread (BP) calculation to previse the expulsion proficiency of CMNs for $\mathrm{Fe}$ (II) particles. Seeking after benchmark correlations of BP calculations, a review was directed to decide the ideal system structure. The yield acquired from the ANN demonstrating was contrasted and the tribulation information. The point of this work is to highlight the likelihood of the prospect of sorption productivity for the metal particles from waste dihydrogen monoxide utilizing CMN in the scope of metal focus with which lab tests have not been led.

*Corresponding author: Mini Namdeo, Centre of Excellence-Nanotechnology Indian Institute of Technology, Roorkee, Uttarakhand, India, Tel: +917060214491; E-mail:mini.namdeo@gmail.com

\section{Received May 12, 2017; Accepted May 15, 2017; Published May 17, 2017}

Citation: Namdeo M, Mehta R, Mehta VK, Agarwala V (2017) Artificial Neural Network Modelling for the Removal of Fe (III) from Aqueous Solutions Using Chitosan Magnetite Nano Composites (CMNs). J Adv Chem Eng 7: 170. doi: 10.4172/2090-4568.1000170

Copyright: (c) 2017 Namdeo M, et al. This is an open-access article distributed under the terms of the Creative Commons Attribution License, which permits unrestricted use, distribution, and reproduction in any medium, provided the original author and source are credited. 


\section{Materials and Methods}

\section{Preparation of Chitosan Magnetite Nano Composites (CMNs)}

Chitosan-magnetite nano composites (CMNs) were set up by synthetic co-precipitation of $\mathrm{Fe}^{2+}$ and $\mathrm{Fe}^{3+}$ particles by $\mathrm{NaOH}$ within the visual perception of chitosan taken after by treatment under aqueous conditions [19]. To a $2 \%$ acidic arrangement of chitosan, iron (II) chloride and iron (III) chloride were broken up in 1:2 molar proportion and the subsequent arrangement was artificially enheartened at $40^{\circ} \mathrm{C}$ by including $30 \% \mathrm{NaOH}$ drop insightful with consistent coalescing, at a controlled $\mathrm{pH}$ (10-10.4). The suspension was warmed at $90^{\circ} \mathrm{C}$ for 1 $\mathrm{h}$ under nonstop commixing and isolated by centrifuging a few times in dihydrogen monoxide and after that in ethanol at $200 \mathrm{rpm}$. The decontamination step was utilized to expel debasements from chitosanmagnetite nano composites (CMN). The particles were at long last dried in vacuum at $70^{\circ} \mathrm{C}$.

\section{Characterization of CMN particles}

The development of nano sized attractive nano composites was affirmed by FTIR, TEM and SAED examination. Figure 1A-1C portrays FTIR spectra of magnetite, chitosan and chitosan-magnetite nano composite $(\mathrm{CMN})$, individually. In the range (A) the nearness of groups in the area $3250-3600 \mathrm{~cm}^{-1}$ and $1550-1700 \mathrm{~cm}^{-1}$ is related with the cross section of water particles, hence demonstrating the nearness of water crystallization in the magnetite test or in $\mathrm{KBr}$. In addition, the trademark crest, exhibit at $610 \mathrm{~cm}^{-1}$ affirms the metal-oxygen extending. The nearness of trademark crest at $3594 \mathrm{~cm}^{-1}$ in the range of chitosan (see Figure 1B) is demonstrative of hydroxyl gatherings while tops showing up at $1650 \mathrm{~cm}^{-1}$ and $1449 \mathrm{~cm}^{-1}$ compare to extending vibrational recurrence of amide I and amide II in chitosan particles. At long last, it can be found in Figure 1C that all these trademark pinnacles are additionally present in range of chitosan-magnetite nano composite.

Figure 2 portrays the TEM picture of chitosan-magnetite nano composite particles alongside SAED particles appeared in inset. The mundane width of particles, as decided from estimation of quantification of 30 particles, culled subjectively from TEM picture, was observed to be virtually $27 \mathrm{~nm}$. It is likewise certain that magnetite particles are consummately inserted inside chitosan particles because of transpiring of two synchronous procedures, one including arrangement

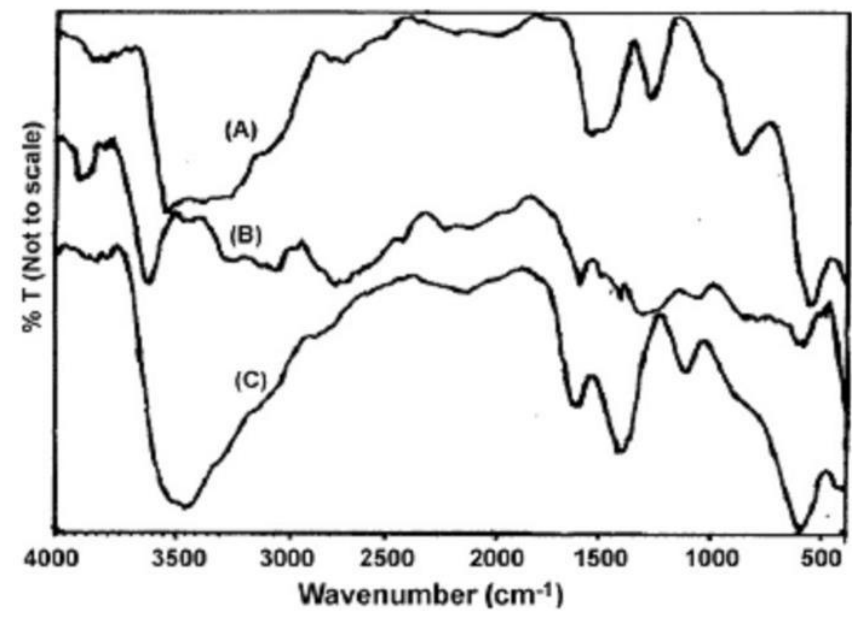

Figure 1: Fourier transform infrared spectra (FTIR) of (A) magnetite, (B) chitosan plain and $(\mathrm{C})$ chitosan magnetite nano composites.

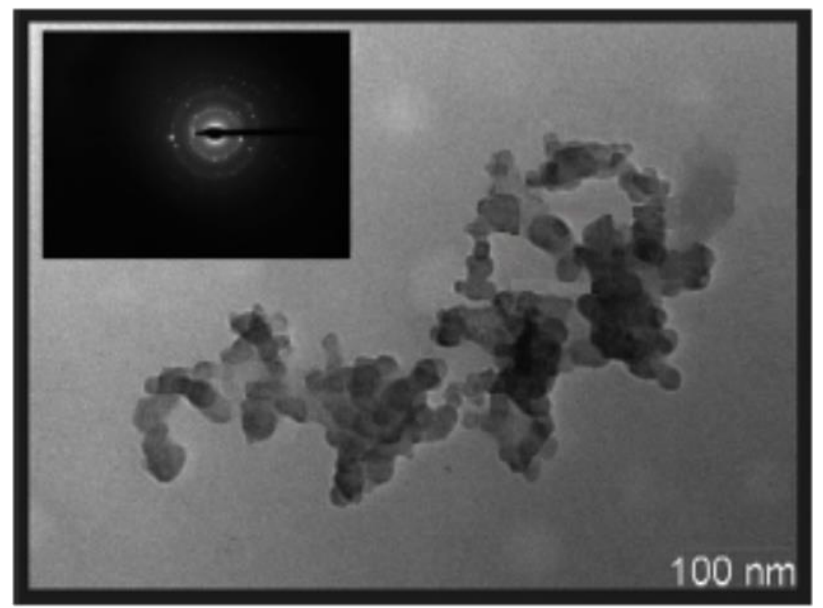

Figure 2: TEM and SAED images of Chitosan magnetite nanocomposites CMN particles.

of magnetite particles and the other including precipitation of chitosan chains in fundamental medium. The headed bolt likewise demonstrates nano composite particles in which both magnetite and chitosan stage are plainly conspicuous. Supplementally, the visually examined agglomerations of nano composite particles may likely be because of the nonappearance of adjustment operator in the replication framework and supplementally because of agglomerating propensity of chitosan. At last, the SAED design (optically discern inset) likewise affirms the arrangement of magnetite nanoparticles.

\section{Fe (II) uptake studies}

Sorption studies using standard practice size of $508 \mu \mathrm{m}$ were carried out in various experiments as a function of sorbent dosage (5.0-60.0 $\mathrm{mg}$ ), volume of the sorbet solution $(20-50 \mathrm{~mL}), \mathrm{Fe}(\mathrm{III})$ concentration $(10-100 \mu \mathrm{g} / \mathrm{mL})$ and contact time (10-150 mins) with two different particle size $(508 \mu \mathrm{m}$ and $84 \mu \mathrm{m})$. The details of methodology have been described in our earlier publications [15]. Per cent metal uptake by the sorbent was computed using the equation:

$$
\% \text { Sorption }=\frac{\mathrm{Co}-\mathrm{Ce}}{\mathrm{Co} \times 100}
$$

Where, $\mathrm{C}_{\mathrm{o}}$ and $\mathrm{C}_{e}$ were the initial and final concentration of metal ions in the solution.

\section{Artificial Neural Network (ANN) model}

Neural Network Toolbox Neuro Solution $5^{\circ}$ scientific programming was utilized to presage the sorption productivity. A few tribulation sets were utilized to build up the ANN demonstrate. A two layer ANN with sigmoid axon exchange capacity was utilized for information and yield layers. The information accumulated from bunch investigations were dissevered into information lattice and sought framework. The two layer sigmoid system verbalizes with utilizable connection amongst sources of info and yield, gave sigmoid layer has enough neurons. LevenbergMarquardt calculation is most expeditious preparing calculation for system of direct size, in this way, utilized as a component of the present work.

\section{Results and Discussion}

\section{Sorption studies}

The sorbet stock arrangement was set up by dissolving a pre-figured amount of $\mathrm{FeCl}_{3}$ in twofold refined water to give last focus $100 \mathrm{mgl}^{-1}$. 
The stock arrangement was enervated to acquire standard arrangements with fixation in the scope of $5-30 \mathrm{mgl}^{-1}$ and their last $\mathrm{pH}$ was transmuted in accordance with 4.5. Fifty millilitres of $\mathrm{FeCl}_{3}$ arrangement of coveted focus was set in a $125 \mathrm{ml}$ Erlenmeyer flagon containing $0.02 \mathrm{~g}$ of CMNs sorbent and was fomented in thermostatic water shower at $50 \mathrm{rpm}$ for $2 \mathrm{~h}$. At the cessation of analysis the sorbent was isolated by filtration and supernatant was impecunious down spectrophotometrically [20].

\section{Optimization of the ANN structure}

Sorption investigations of Fe (III) particles utilizing chitosan magnetite nano composites was figured by lab cluster tries as a component of contact time, metal particle focus sorbet measurement, and volume of sorbet arrangement, as far as rate expulsion. ANN show in light of two layered discontinuous back inciting estimation for the exploratory data, created from the above bundle examinations was associated with set up the neural framework. In the midst of setting up, the yield vector is handled by a forward go in which the data is spread forward through the framework to figure the yield estimation of each unit. The yield vector is then differentiated and the pined for vector which come to fruition into mix up movement for each yield unit. Remembering the true objective to restrict the oversight, legitimate alterations were made for each of the weights of the framework. After a couple of such cycles, the framework was set up to give the pined for yield for a given data vector. The two layers compose structure included ten neurons for both the layers, portraying the movement of Fe (III) in spouting (Figure 3 ). The execution of system reproduction was assessed as far as mean square blunder (MSE) standard. The created organize model was analysed for its capacity to anticipate the reaction of trial information with the preparation program.

\section{Sensitivity analysis}

An affectability examination was directed to decide the level of viability of factors. Execution of the gatherings of info vectors included contact time, metal particle focus sorbet measurements, and volume of sorbet arrangement. Every one of the models e length of information was appropriated as $60 \%$ for preparing, $25 \%$ for approval and $15 \%$ for the testing. Then again Two Layer models are produced in which initially layer has 10 neurons and the quantities of concealed neuron are additionally 10. For preparing calculation Levenberg-Marquardt is utilized to prepare the model. Weights have been upgraded as for least mistake.

\section{Effect of contact time}

The vitality of sorption process portrays the solute take-up, which, along these lines directs the home time of the sorption reaction.

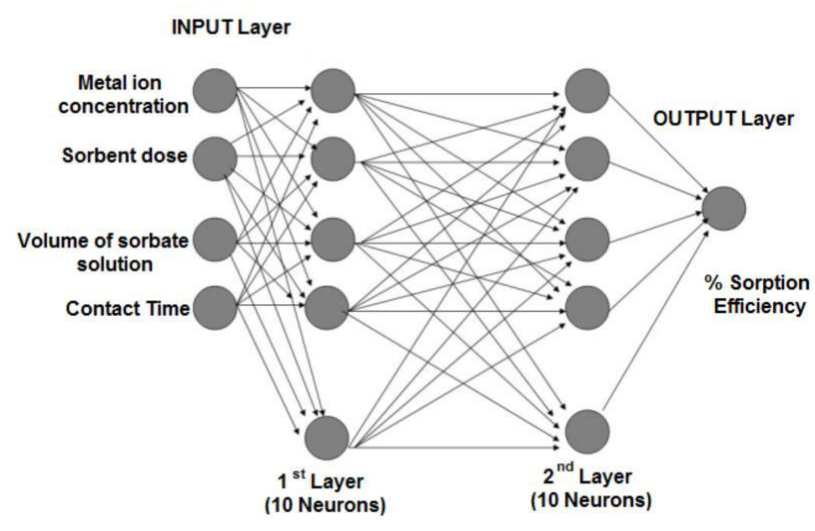

Figure 3: Two Layer Optimum Neural Network structure for the Sorption efficiency.
Remembering the true objective to understand the engine perspectives for Fe (III) take-up on CMNs, the sorption vitality was finished with sorbent particles of two exceptional sizes specifically $508 \mu \mathrm{m}$ and 84 $\mu \mathrm{m}$, at room temperature for the basic Fe (III) obsession $20 \mathrm{mgl}^{-1}$. Clearly measure of Fe (III) sorbed augmentations with time, and more diminutive size particles show higher Fe (III) take-up. This may simply be credited to the way that particles with tinier size have greater surface range, subsequently giving all the additionally limiting goals to iron take-up. Figure 4 exhibits the result gotten by the neural framework generation for the arrangement, cross endorsement and testing enlightening files on effect of contact time. Best endorsement execution was seen to be 3.279 at age 8 . While, Figure 5 addresses the examination of trial data and ANN yields for Fe (III) molecule at effect of contact time, suggests that the ANN show performed well for gauge of the test data.

In Figure 6 the blue bars speak to preparing information, the green bars speak to approval information, and the red bars speak to testing information. The histogram can give you a sign of exceptions, which are information focuses where the fit is fundamentally more awful than the dominant part of information. In this plot a large portion of mistakes are fall between 4 to 2.5 . This blunder histogram graphically speaks to the dispersion of information and a plot of the quantity of information focuses in every 20 canister. Exploratory histogram delineates zero mistakes after rehashed trials of 20 examples. These reviews presume that ANN approach is very proficient in displaying complex adsorption marvel. The blunder histogram is a plot to get extra confirmation of system execution.

\section{Effect of Fe (III) concentration}

The measure of $\mathrm{Fe}$ (III) sorbed per gram of sorbent (i.e., $\mathrm{x} / \mathrm{m}$ ) was found to vary in interesting way with starting groupings of sorbet plan in the range $20-360 \mathrm{mgl}^{-1}$. The results exhibits that $\mathrm{x} / \mathrm{m}$ increases with beginning $\mathrm{Fe}$ (III) obsessions up to $160 \mathrm{mgl}^{-1}$ and a while later it demonstrates lessening design with further augmentation in sorbet centres. The basic addition in $\mathrm{x} / \mathrm{m}$ may be credited to the route that with augmentation in $\mathrm{Fe}$ (III) obsession, more metal molecule sorbed into the CMN particles. Regardless, past $160 \mathrm{mg} \mathrm{l}^{-1}$ concentration of $\mathrm{Fe}$ (III) the decrease in $\mathrm{x} / \mathrm{m}$ may be a result of deterioration of chitosan from CMN particles as the $\mathrm{pH}$ of the sorbet plan was seen to be in the range 2-3. Since chitosan is dissolvable in acidic $\mathrm{pH}$, its deterioration from the $\mathrm{CMN}$ particles achieved reduction in Fe (III) take-up. Figure 7 shows that the result obtained by the neural framework diversion for the arrangement, cross endorsement and testing enlightening accumulations on effect of Fe (III) obsession. Best Validation Performance was seen to be 48.8226 at age 5. The trial data and ANN yields for Fe (III) particles centres (Figure 8) exhibits a better than average comprehension. The ANN demonstrates performed well for figure of the trial data. Figure 9 plot of mistake histogram graphically speaks to the circulation of information and various information focuses in every 20 receptacle. Exploratory histogram portrays zero mistakes after rehashed trials of 12 occurrences. These reviews presume that ANN approach is very productive in demonstrating complex adsorption wonder. The mistake histogram is a plot to get extra confirmation of system execution. The histogram can give you a sign of anomalies, which are information focuses where the fit is essentially more awful than the greater part of information. In this plot the greater part of blunders are fall between 64 to 5 .

\section{Effect of sorbent dose}

The sorbet arrangement of distinct focus $50 \mathrm{mg} \mathrm{l}^{-1}$ was upset with various amounts of sorbent, in the range 5-60 mg for every $50 \mathrm{ml}$ and 


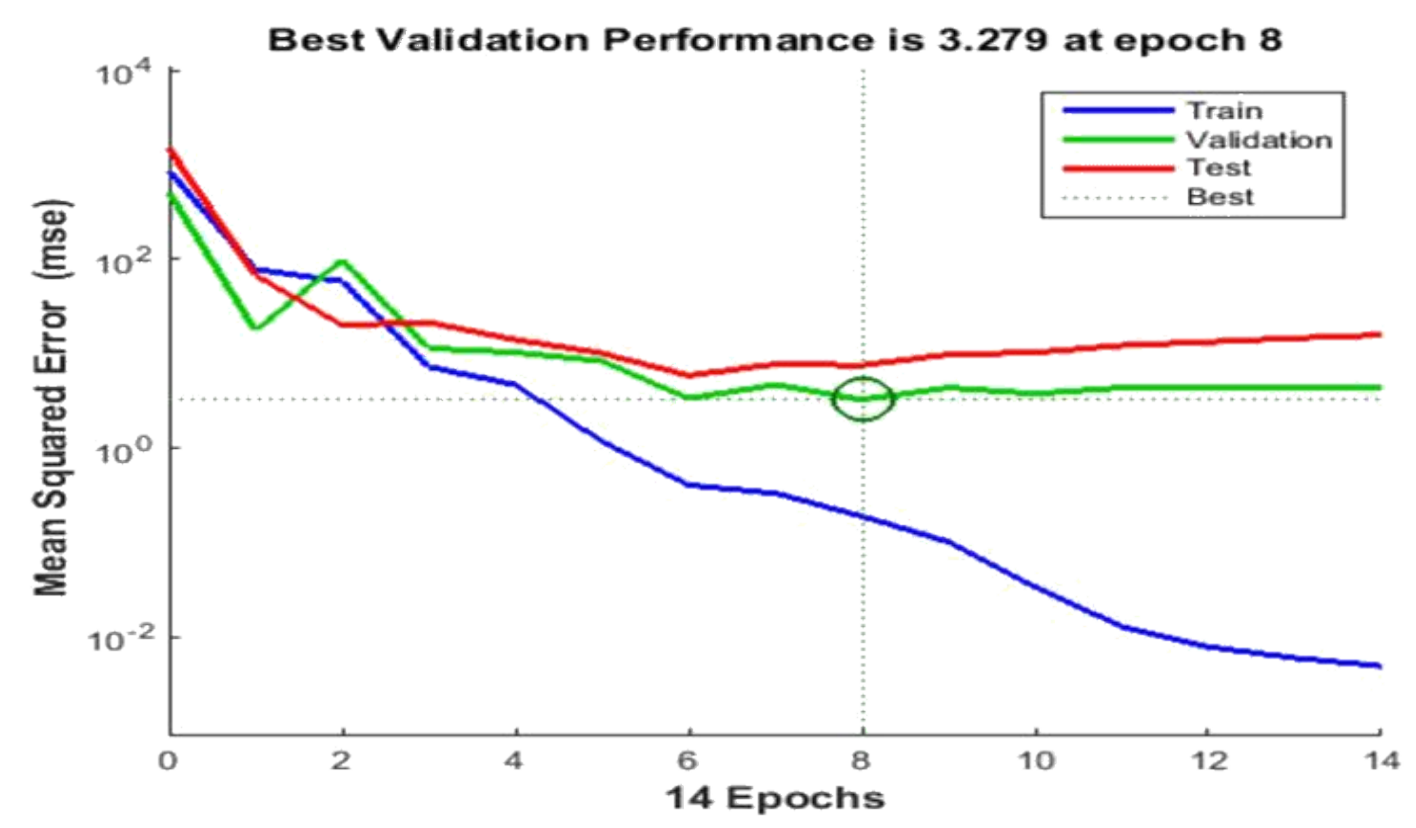

Figure 4: Graphical representation of Mean square error (MSE) value for contact time.

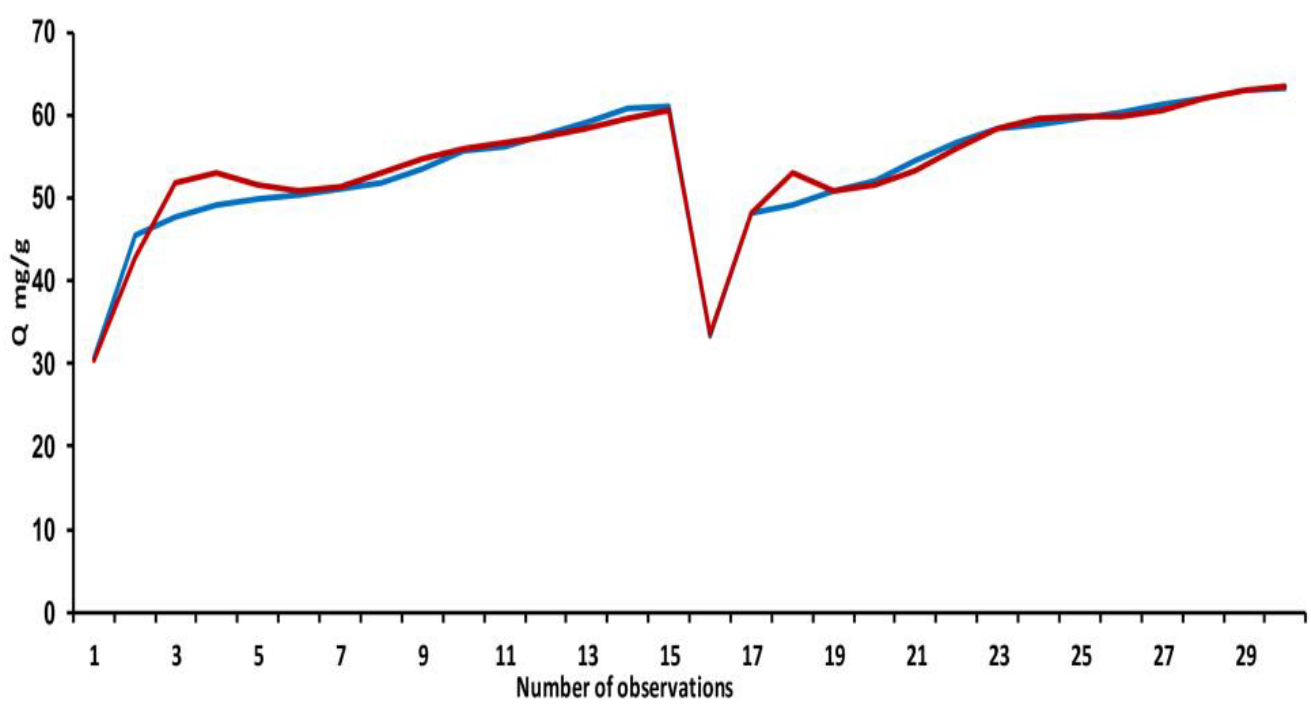

$$
\text { —_OBSERVED —Q-MODELED }
$$

Figure 5: ANN predicted plot versus Fe (III) ions removal by CMN particles at contact time of 150 minutes.

the arrangements were broke down for per cent Fe (III) take-up and $\mathrm{x} / \mathrm{m}$ (i.e., press sorbed in $\mathrm{mg}$ for $1 \mathrm{~g}$ of sorbent). The outcomes delineate that $\%$ sorption and $\mathrm{x} / \mathrm{m}$ values appear inverse patterns. The watched increment in \% sorption with sorbent dosage can be basically ascribed to the way that expansion in measure of sorbent result in increment in surface-range therefore giving an ever increasing number of restricting destinations for $\mathrm{Fe}$ (III) take-up. This at long last outcomes in improvement in per cent take-up. In any case, the diminishing in the estimation of $\mathrm{x} / \mathrm{m}$ with sorbent measurement is because of the way that with the expansion in sorbent dosage from $5 \mathrm{mg}$ to $60 \mathrm{mg}$, the \% take-up increments from 81 to 94 . Figure 10 demonstrates the outcome acquired by the neural system recreation for the preparation, cross approval and testing informational collections on impact of sorbent measurements. Best Validation Performance is observed to be 1.5796 up to fourth decimal place at age 1. A scrutiny of test information and ANN yields as an element of sorbent dose (Figure 11) delineated execution of the model in great congruity with the trial information.

Figure 12 plot of mistake histogram graphically speaks to the conveyance of information and various information focuses in every 20 canister. Exploratory histogram portrays zero blunders after rehashed trials of 8 occurrences. These reviews infer that ANN approach is very productive in demonstrating complex adsorption wonder. The blunder histogram is a plot to acquire extra check of system execution. The 


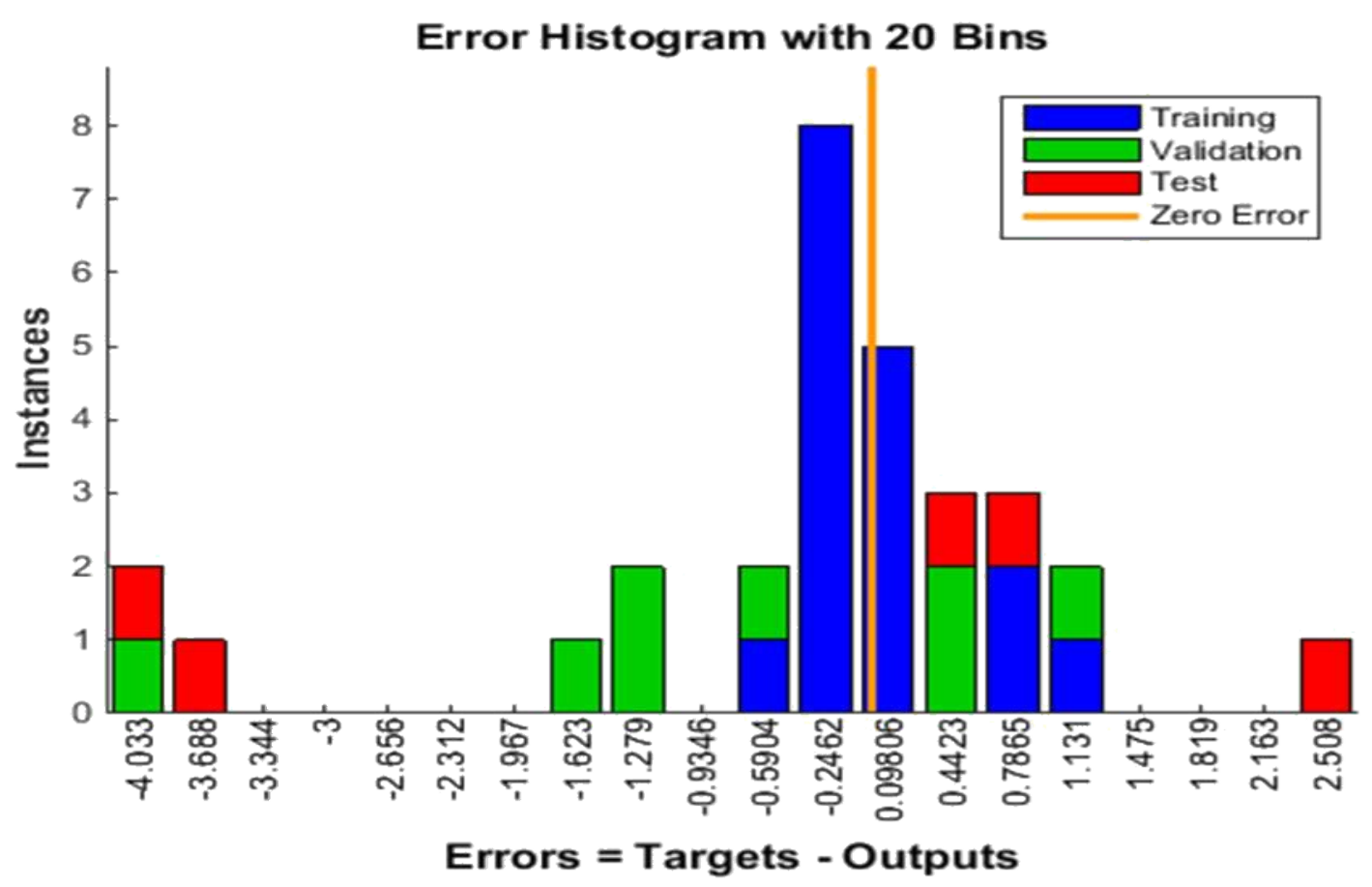

Figure 6: Error histogram plot for trained ANN on Effect of Contact time.

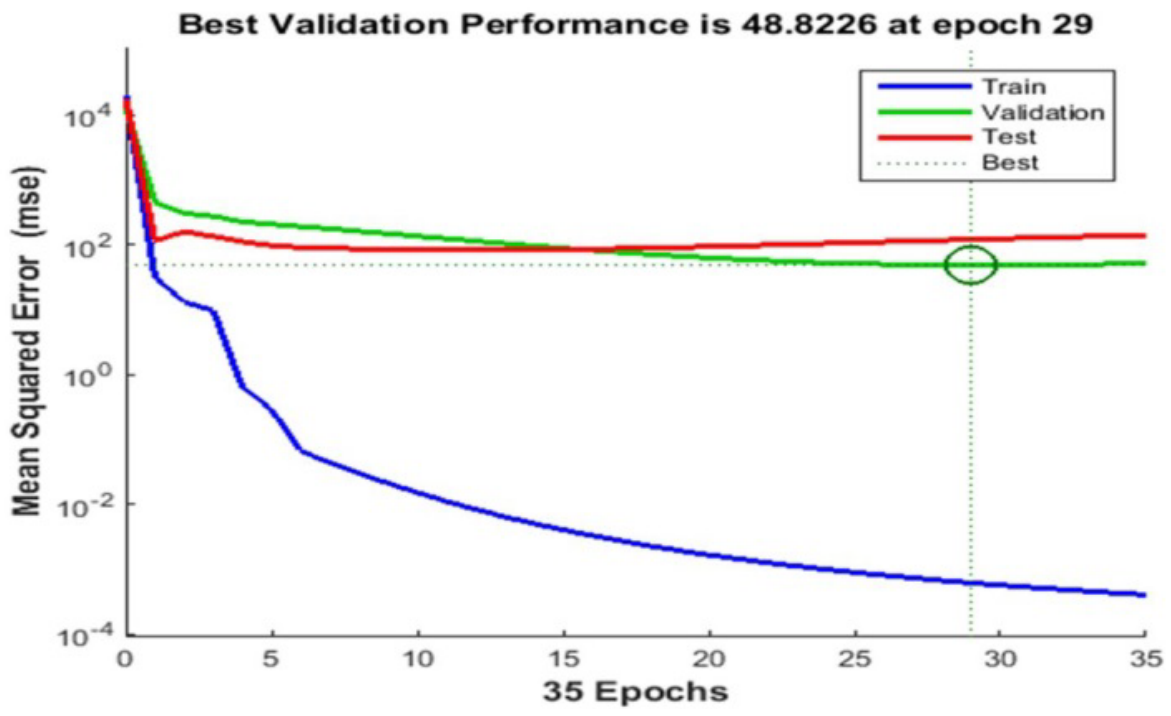

Figure 7: Graphical representation of MSE value at different Fe (III) concentrations.

histogram can give you a sign of anomalies, which are information focuses where the fit is essentially more terrible than the lion's share of information. In this plot the greater part of blunders are fall between 0.85 to 2 .

\section{Effect of chitosan content}

The assortment in joining of chitosan in nano composites is required to realize an alteration in sorption direct of Fe (III). To assess this, chitosan-magnetite nano composites with different substance of chitosan were consolidated by moving the measure of chitosan in the sustain mix in the range $0.5-5.0 \%$. The results, exhibit that as the measure of chitosan is extended in nano composite, $\mathrm{Fe}$ (III) also augments. This may be illuminated on the start of the way that the higher gathering of chitosan gives furthermore limiting regions to sorption and consequently the take-up of Fe (III) augmentations. It is also worth determining that as the chitosan obsession is extended past $4 \%$, every single dynamic goal are inundated in this way Fe (III)- takeup fulfils perfect regard.

Figure 13 exhibits the result gotten by the neural framework propagation for the arrangement, cross endorsement and testing enlightening lists on effect of chitosan substance. Best Validation Performance is seen to be 0.17962 up to fifth decimal place at age 5 . 


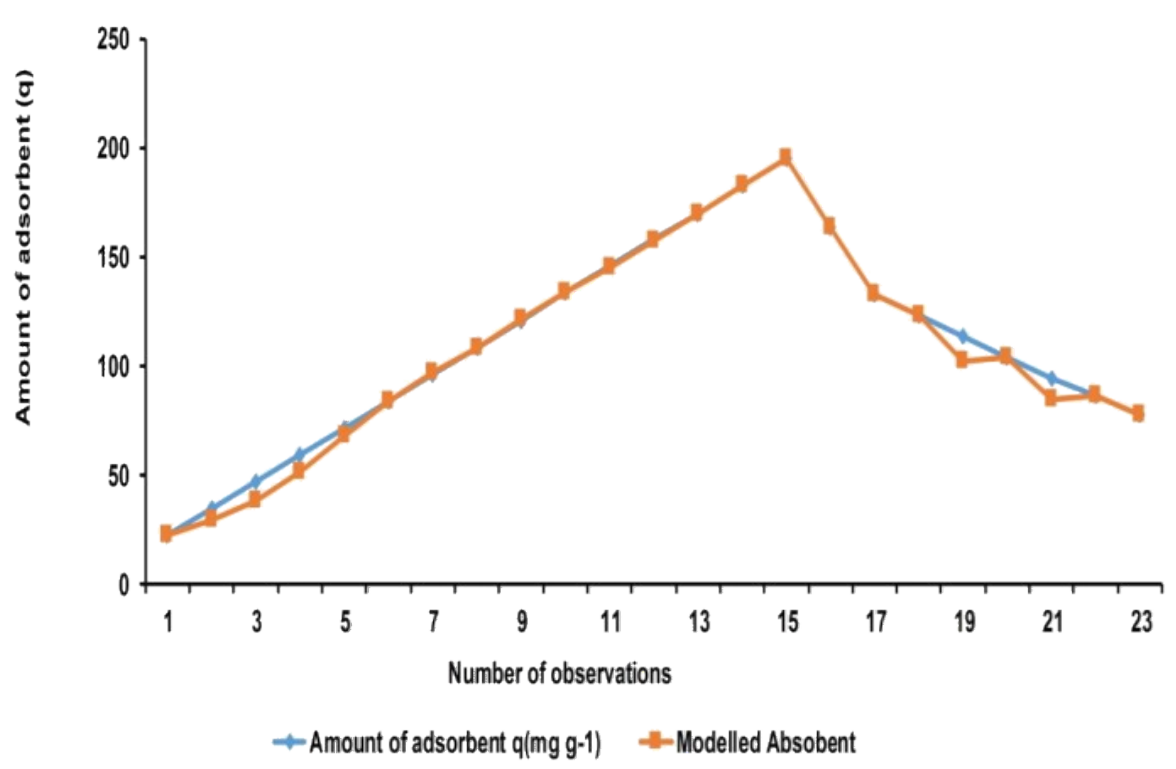

Figure 8: Plot of the ANN predicted versus effect of per cent removal of Fe (III) by CMNs.

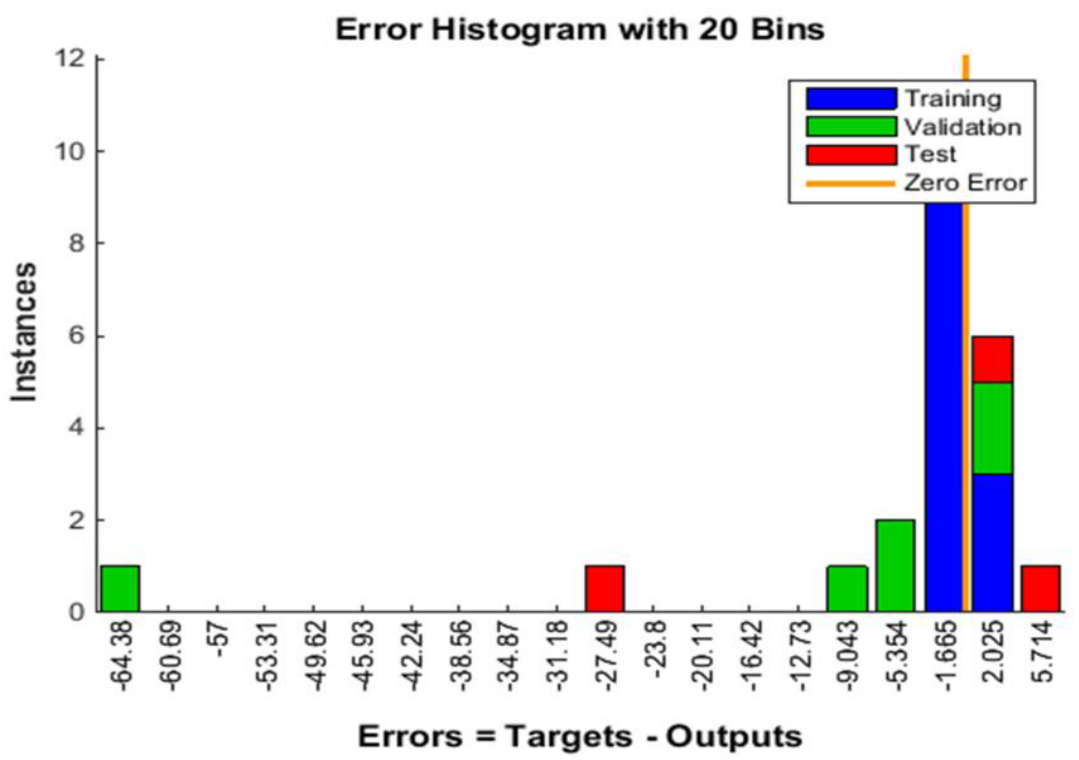

Figure 9: Error histogram plot for trained ANN on Effect of Fe (III) concentration.

ANN exhibit demonstrated execution in resemblance with exploratory data (Figure 14).

Table 1 compiles all the values of MSE and $\mathrm{R}^{2}$ for different effect which we have discussed in present work. Figure 15 plot of blunder histogram graphically speaks to the dispersion of information and various information focuses in every 20 canister. Exploratory histogram delineates zero blunders after rehashed trials of 11 cases. These reviews presume that $\mathrm{ANN}$ approach is very effective in demonstrating complex adsorption wonder. The blunder histogram is a plot to get extra confirmation of system execution. The histogram can give you a sign of anomalies, which are information focuses where the fit is fundamentally more regrettable than the larger part of information. In this plot the vast majority of mistakes are fall between 0.73 to 0.65 . From the above review it might be presumed that chitosan-magnetite nano composites (CMNs) are successful sorbent for evacuation of Fe (III) from fluid arrangement The two layer ANN displaying strategy is permitted to streamline this procedure. The Levenberg-Marquardt calculation (LMA) was discovered best of BP calculations with a base estimation of mean squared blunder (MSE) for preparing and cross approval. In this manner a straightforward back engendering of the repetitive system utilizing the energy preparing calculation is demonstrated significant supplement for the traditional and convoluted numerical models in the expectation of bioprocess Introduction of learning based frameworks is effective for logical research of unstudied reliance among various by characteristic factors (physical, substance, organic) for unravelling undertakings of inferential estimation and process enhancement without requiring a major measure of exact exploratory information. 


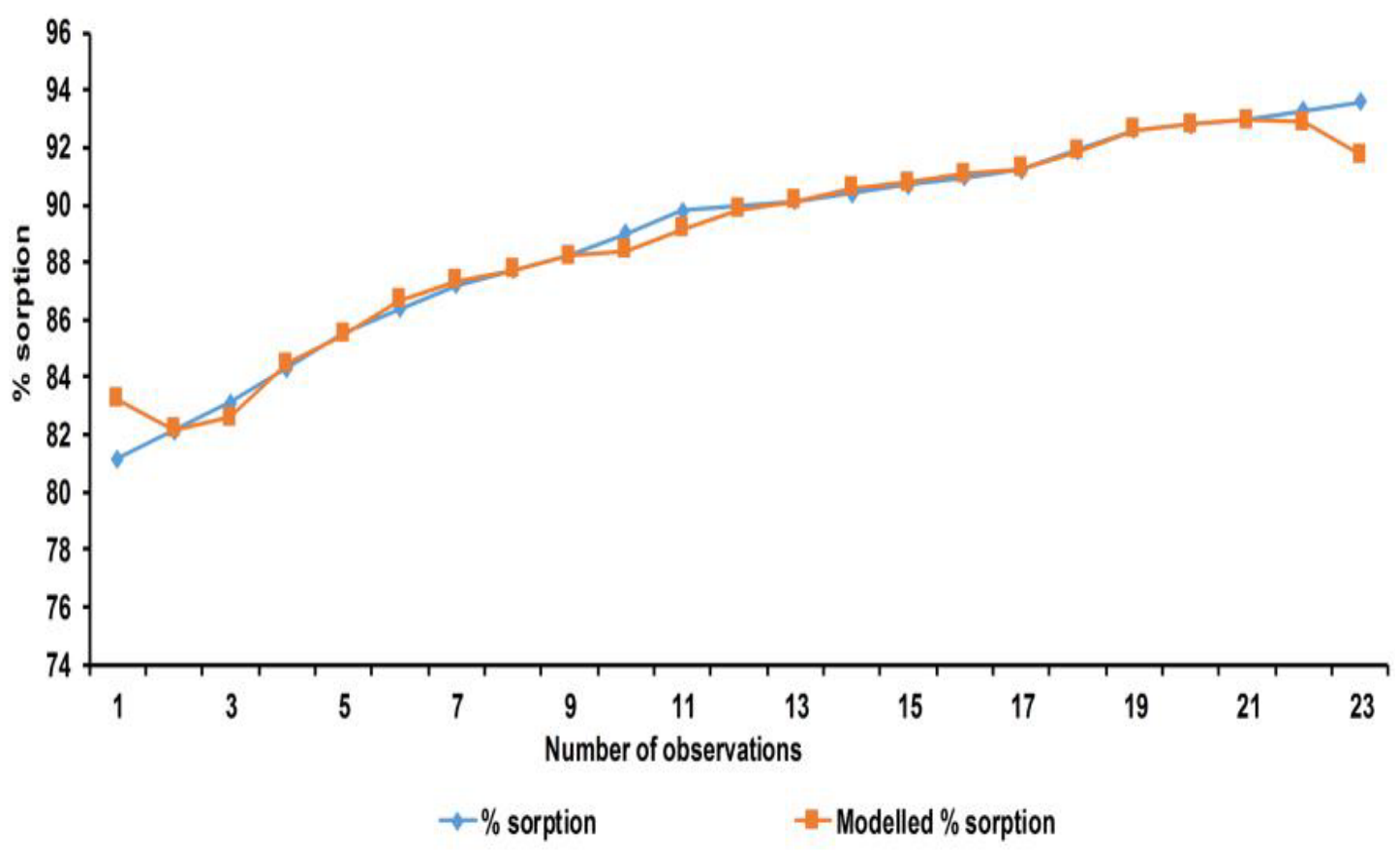

Figure 10: Graphical representation of MSE value for sorbent dose.

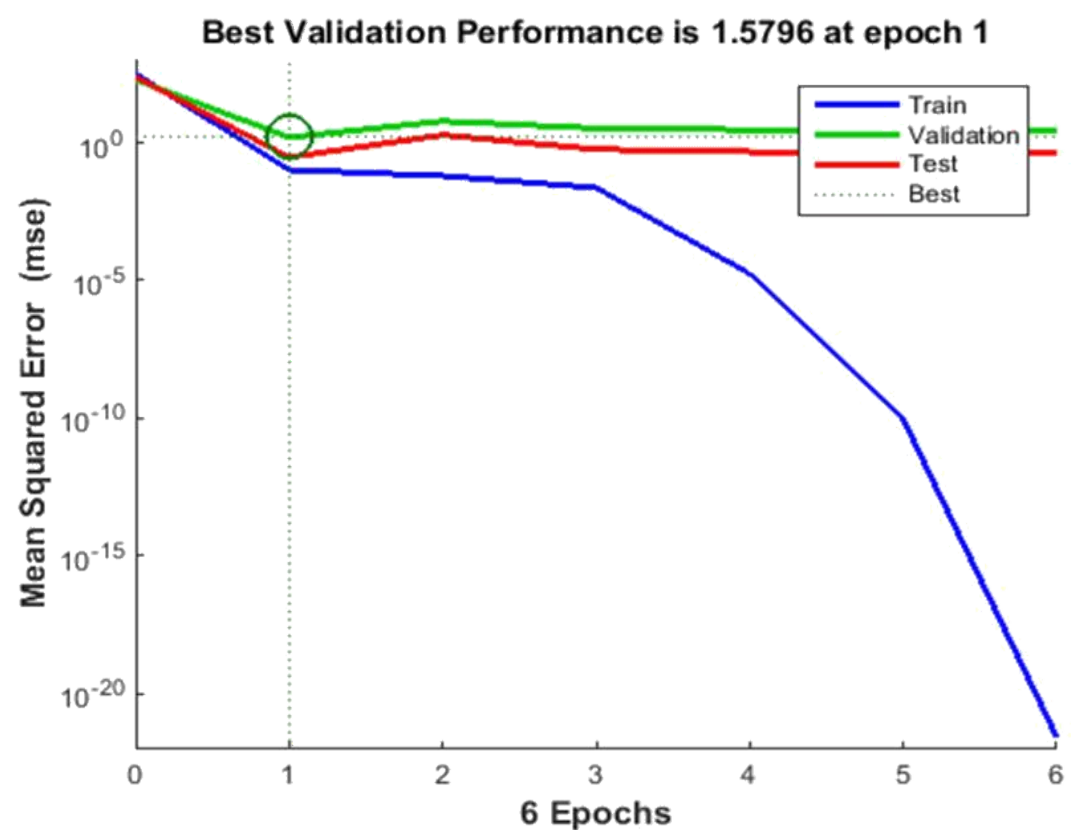

Figure 11: Acquiescent between ANN outputs and experimental data as a function of sorbent dosage.

\begin{tabular}{|c|c|c|c|c|c|c|c|}
\hline \multirow{2}{*}{ Input data for ANN Model } & \multicolumn{3}{|c|}{ Mean Square Error (MSE) Values } & \multicolumn{4}{|c|}{ Regression Values $\left(\mathbf{R}^{2}\right)$} \\
\hline & Training & Validation & Testing & Training & Validation & Testing & All \\
\hline Effect of Con tact Time & 0.191067 & $327 S 99$ & $7.528 \mathrm{~S}$ & 0.99891 & 0.96179 & $0 . S 0247$ & 0.98182 \\
\hline Effect of Fe (III) Concentration & 0.700712 & 4S.S2255 & 121.1399 & 0.999854 & $0 . S 1286 S$ & 0.99987 & 0.95144 \\
\hline Effect of Sorbent Dose & 0.3199 & 0.043667 & 1.553 & $0.9 S 8276$ & $0.99 S 665$ & 0.95986 & $0.9564 S$ \\
\hline Effect of Chitosan Content & $3.439 \mathrm{E}-25$ & 0.1796 IS & $0.29802 S$ & 1 & 0.9992 & 0.99719 & $0.9994 S$ \\
\hline
\end{tabular}

Table 1: Shows Performance Indices values of different effects. 


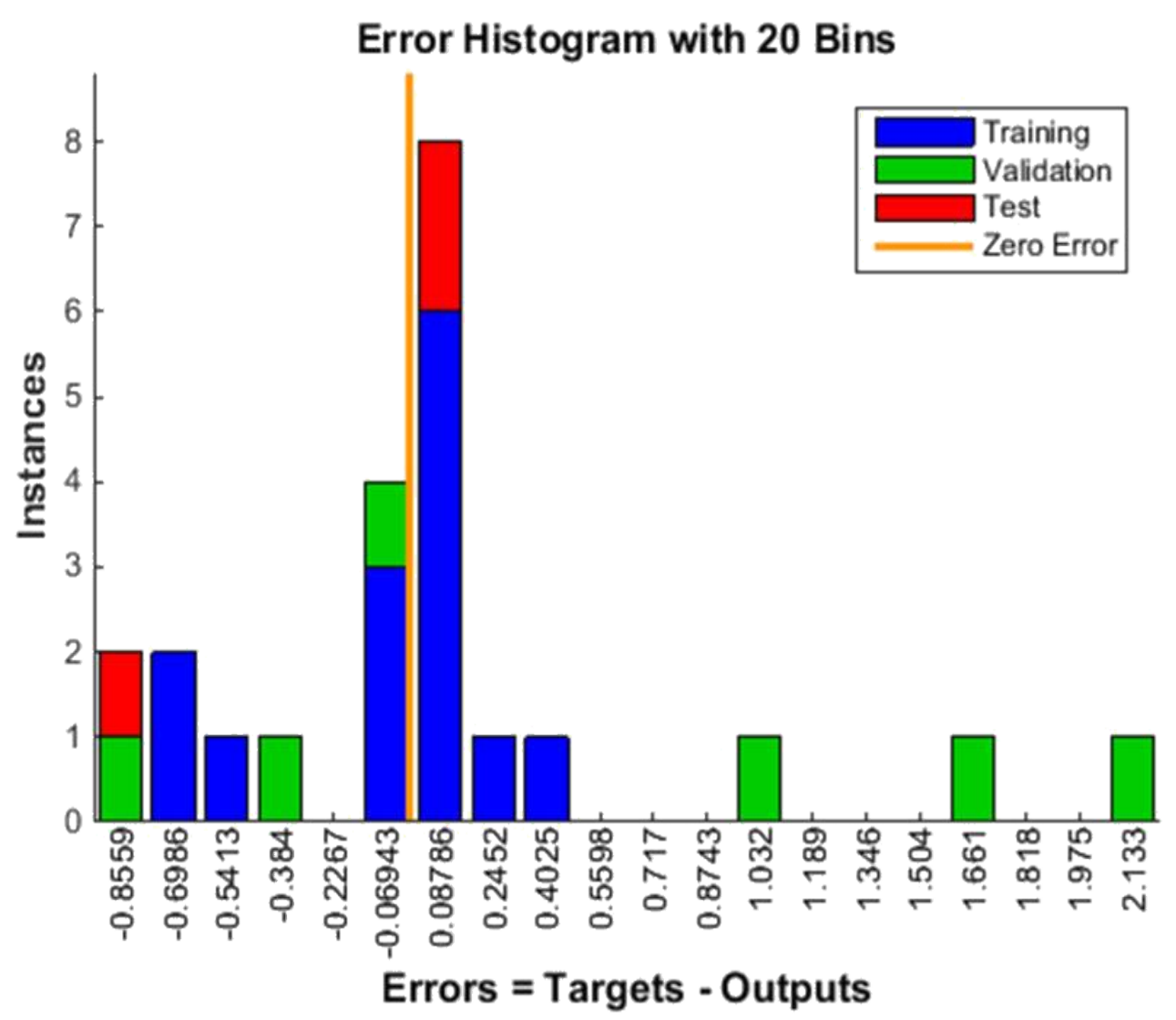

Figure 12: Error histogram plot for trained ANN on Effect of Fe (III) concentration.

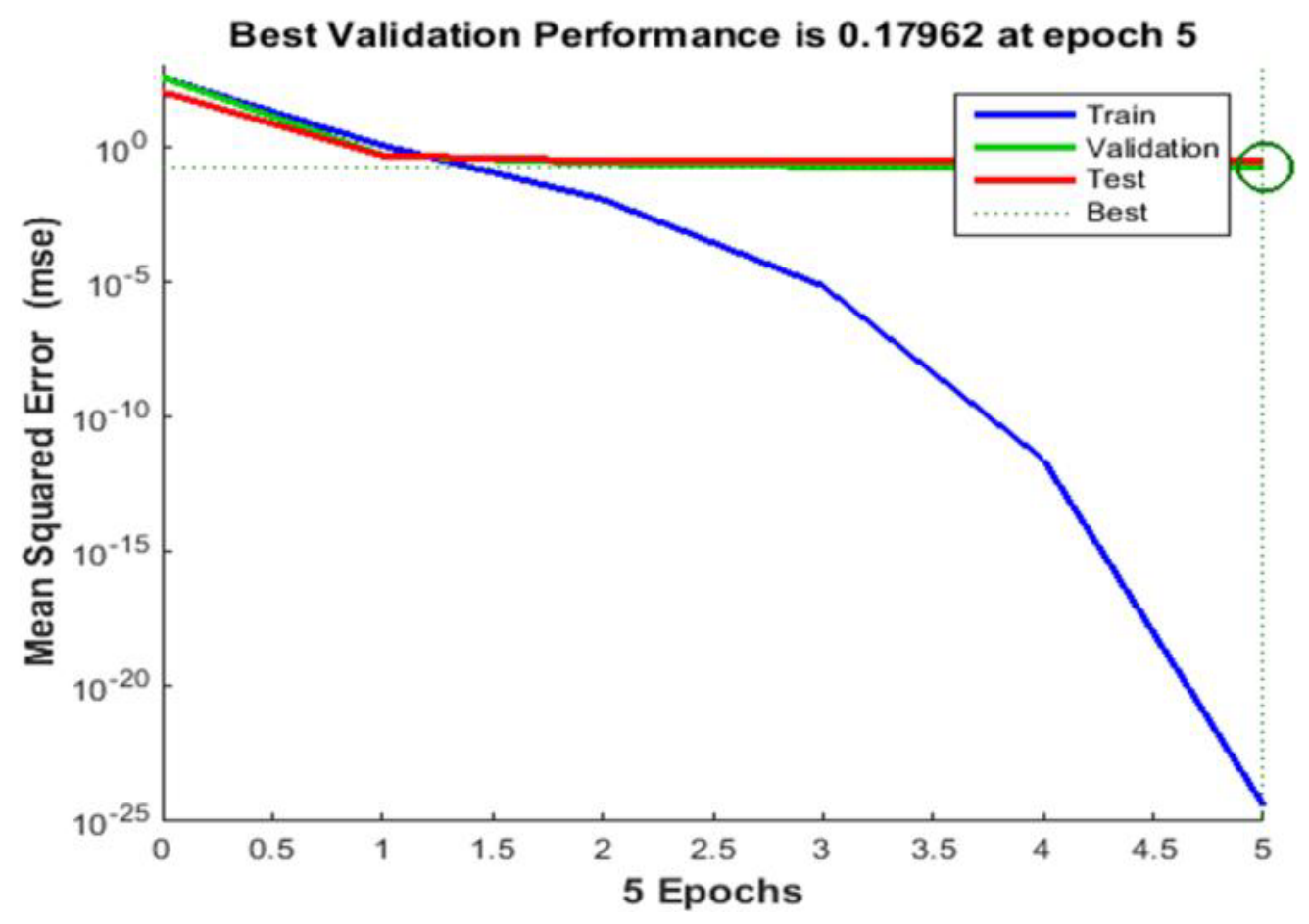

Figure 13: Graphical portrayal of MSE incentive with 0.17962 at age 5 . 


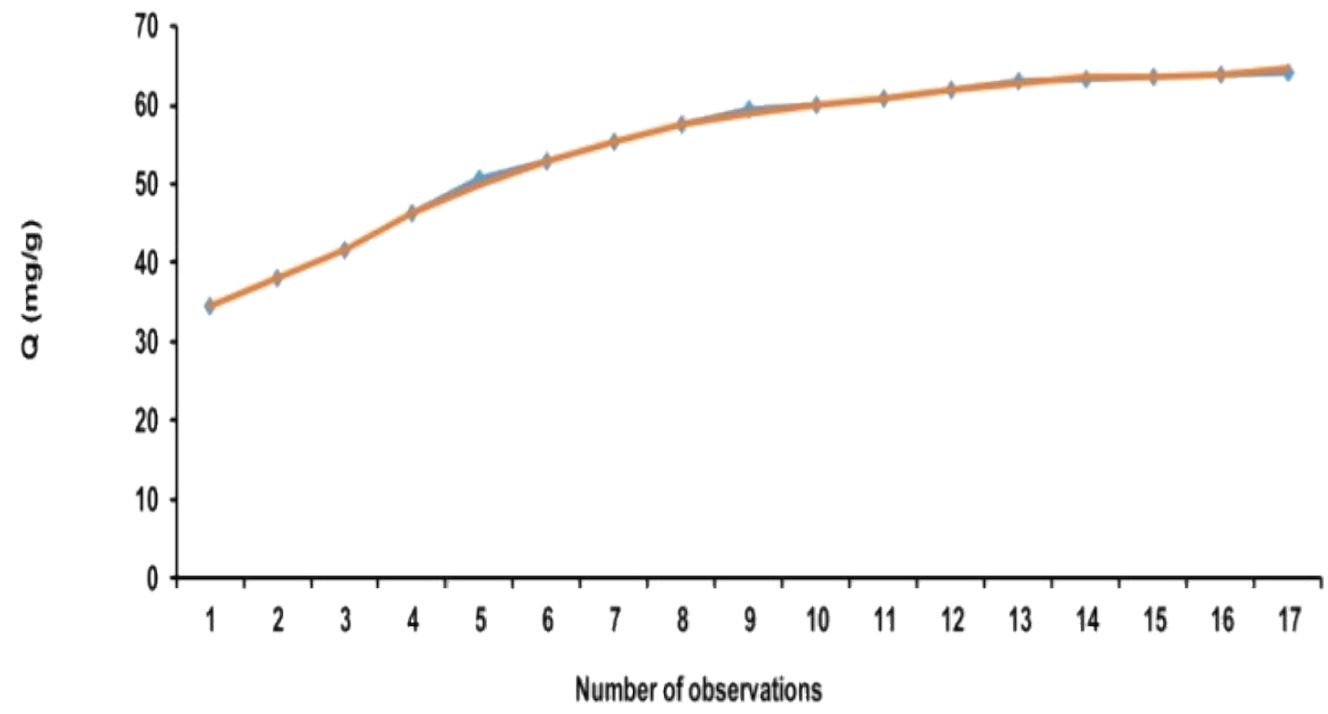

$\rightarrow q(m g g-1) \quad-$ Modelled $Q$

Figure 14: Agreement between ANN outputs and experimental data as a function of chitosan content.

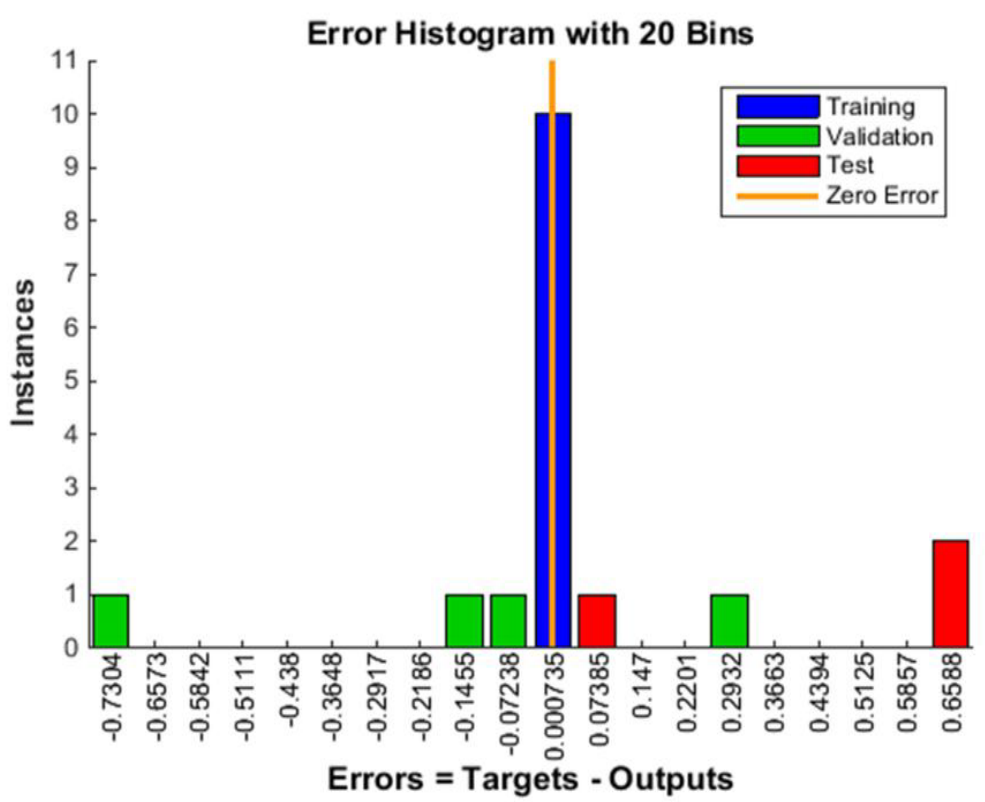

Figure 15: Error histogram plot for trained ANN on Effect of Fe (III) concentration.

\section{Conclusions}

From the above survey it may be deduced that chitosan-magnetite nano composites (CMNs) are convincing sorbent for ejection of Fe (III) from watery game plan the two layers ANN showing framework is allowed to enhance this method. The Levenberg Marquardt count (LMA) was found best of BP computations with a base estimation of mean squared error (MSE) for planning and cross endorsement. Thus a direct back spread of the irregular framework using the vitality get ready computation is exhibited imperative supplement for the general and jumbled numerical models in the desire of bioprocess Introduction of learning based structures is capable for legitimate research of unstudied dependence among different by ordinary components (physical, substance, natural) for clarifying endeavours of inferential estimation and process improvement without requiring a noteworthy measure of correct exploratory data.

\section{Acknowledgement}

The authors gratefully acknowledge Prof. S. K. Nath Head, Centre of Excellence Nanotechnology, Indian Institute of Technology, Roorkee, and Dr. R.D Singh, Director, National Institute of Hydrology, Roorkee, Uttarakhand, India for their support and providing facilities. Authors also would like pay our last respect towards Late Prof Vijaya Agrawala, for being continuous motivation and inspiration throughout the conduction of this work. 
Citation: Namdeo M, Mehta R, Mehta VK, Agarwala V (2017) Artificial Neural Network Modelling for the Removal of Fe (III) from Aqueous Solutions Using Chitosan Magnetite Nano Composites (CMNs). J Adv Chem Eng 7: 170. doi: 10.4172/2090-4568.1000170

\section{References}

1. Champan H (1995) Iron Nutrition and Physiological Significance. The British Nutrition Foundation.

2. Lag J (1993) Geomedical, Problems Related to Aluminum, Iron and Magnesium. The Norwegian Academy Science and Letter.

3. Lauffer R (1992) Iron and Human Diseases. CRC Press, London.

4. Bunei R, Kawasaki N, Ogata F, Nakamura T, Aochi K, et al. (2006) Removal of lead and iron ions by vegetable biomass in drinking water. J Oleo Sci 55: 423-427.

5. Burke EY, Hasirci N, Yilmaz O (2002) Iron (III) ion removal from solution through adsorption onto chitosan. J Appl Polym Sci 84: 1185-1192.

6. Nakajima T, Sakaguchi S (2000) Uptake and removal of iron by immobilized persimmon tannin. J Chem Technol Biotechnol 75: 977-982.

7. Nassar MM, Ewida KT, Ebrahiem EE, Magdy YH, Mheadi MH (2004) Adsorption of iron and manganese using low cost materials as adsorbents. J Environ Sci Health: Part A 39: 421-434.

8. Sirichote O, Innajitara W, Chuenchom L, Chuchit D, Sangklanakarin N (2002) Adsorption of iron (III) ion on activated carbons obtained from bagasse, pericarp of rubber fruit and coconut shell. J Sci Technol 24: 235-242.

9. Lee MS, Nicol MJ (2007) Removal of iron from cobalt sulfate solutions by ion exchange with Diphonix resin and enhancement of iron elution with titanium (III). Hydrometallurgy 86: 6-12.

10. Karthikeyan G, Muthulakshmi N, Andal Anbalagan K (2005) Adsorption studies of iron (III) on chitin. J Chem Sci 117: 663-672.
11. Schmuhl R, Krieg HM, Keizer K (2001) Adsorption of $\mathrm{Cu}$ (II) and $\mathrm{Cr}(\mathrm{VI})$ ions by chitosan: kinetics and equilibrium studies. Water SA 27: 1-8.

12. Yi H, Wu L, Bentley WE, Ghodssi R, Rubloff GW, et al. (2005) Bio fabrication with chitosan. Biomacromolecules 9: 2881-2894.

13. Nomanbhay SM, Palanisamy K (2005) Removal of heavy metal from industrial waste water using chitosan coated oil palm shell charcoal. Electron J Biotechnol 8: 44-53.

14. Moeser GD, Roach KA, Green WH, Laibinis PE, Hatton TA (2002) Water-based magnetite fluids as extrants for synthetic organic compounds. Ind Eng Chem Res 41: 4739.

15. Namdeo M, Bajpai SK (2008) Chitosan-magnetite nano composites (CMNs) as magnetic carrier particles for removal of $\mathrm{Fe}$ (III) from aqueous solutions. Colloids and Surfaces A: Physicochem Eng Aspects 320: 161-168.

16. Park YS, Chon TS, Kwak IS, Lek S (2004) Hierar-chical Community Classification and Assessment of Aquatic Ecosystems Using Artificial Neural Networks. Science of the Total Environment 327: 105-122.

17. Belanche L, Valdes JJ, Comas J, Roda IR, Poch M (2000) Prediction of the Bulking Phenomenon in Waste-water Treatment Plants. Artificial Intelligence in Engi-neering 14: 307-317.

18. Shetty GR, Chellam S (2003) Predicting Membrane Fouling during Municipal Drinking Water Nanofiltration Using Artificial Neural Networks. Journal of Membrane Science 217: 69-86.

19. Huang SH, Liao MH, Chen DH (2003) Direct binning and characterization of lipase onto magnetite nano-particle. Biotechnol Prog 19: 1095-1100.

20. APHA (1989) Standard Methods for examination of Water and Waste Water 17th edn. American Public Health Association, New York, USA. 